NASA Technical Memorandum 107099

\title{
A Compact Fiber Optic Eye Diagnostic System
}

Rafat R. Ansari and Kwang I. Suh

Lewis Research Center

Cleveland, Ohio

Stephen Dubin and Michael A. DellaVecchia

Drexel University

Philadelphia, Pennsylvania

Prepared for the

Space Technology and Applications International Forum

sponsored by the NASA Technology Utilization Foundation

Albuquerque, New Mexico, January 7-11, 1996

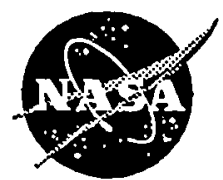

National Aeronautics and

Space Administration

\section{LIBRARY COPY}

JAN 101996

LANGLEY RESEARCH CENTER LIBRARY NASA HAMPTON, VIRGINIA 


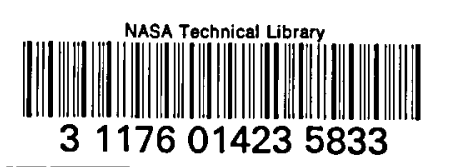

\title{
A COMPACT FIBER OPTIC EYE DIAGNOSTIC SYSTEM
}

\author{
Rafat R. Ansari and Kwang I. Suh* \\ NASA Lewis Research Center \\ Mail Stop 333-1, 21000 Brookpark Road \\ Cleveland, Ohio 44135 \\ Phone: (216) 433-5008, Fax: (216) 977-7138 \\ e-mail: rra@po.cwru.edu
}

\author{
Stephen Dubin and Michael A. DellaVecchia \\ Biomedical Engineering and Science Institute \\ Drexel University \\ Philadelphia, Pennsylvania 19104 \\ Phone: (215) 895-2219, Fax: (215) 895-4983 \\ e-mail: dubinse@duvm.ocs.drexel.edu
}

\begin{abstract}
$\underline{\text { Abstract }}$
A new fiber optic probe developed for determining transport properties of sub-micron particles in fluids experiments in a microgravity environment has been applied to study different parts of an eye. The probe positioned in front of an eye, delivers a low power $(\sim$ few $\mu \mathrm{W})$ light from a laser diode into the eye and guides the light which is back scattered by different components (aqueous humor, lens, and vitreous humor) of the eye through a receiving optical fiber to a photo detector. The probe provides rapid determination of macromolecular diffusivities and their respective size distributions in the eye lens and the gel-like material in the vitreous humor. In a clinical setting, the probe can be mounted on a standard slit-lamp apparatus simply using a Hruby lens holder. The capability of detecting cataracts, both nuclear and cortical, in their early stages of formation, in a non invasive and quantitative fashion, has the potential in patient monitoring and in developing and testing new drugs or diet therapies to "dissolve" or slow down the cataract formation before the surgery becomes necessary. The ability to detect biochemical and macromolecular changes in the vitreous structure can be very useful in identifying certain diseases of the posterior chamber and their complications, e.g., posterior vitreous detachment and diabetic retinopathy.
\end{abstract}

\section{INTRODUCTION}

In a normal eye the anterior chamber (space between the cornea and the lens) of the eye is filled with a transparent water-like fluid known as aqueous humor. However, presence of elevated cholesterol and blood-sugar levels in patients with heart and diabetic related diseases can be identified in this fluid. An adult human lens is the size of an aspirin tablet. At birth the lens tissue is transparent. With age the lens gradually loses its transparency. The lens transparency can also be lost because of trauma, certain diseases (e.g. diabetes, uremia, chronic diarrhea), hereditary factors, radiation exposure, and continued use of certain medications over a long period of time including steroids, tranquilizers (phenothiazines), gout medications (allopurinol), psoriasis medications (psoralens), and antibiotics (tetracycline). A cataract is formed when the lens cloudiness hinders light transmission through the lens and the ability to focus a sharp image on the retina. Cataracts remain the major cause of blindness affecting about 50 million people each year worldwide. It is estimated that over $\$ 5$ billion will be spent this year in treating cataract patients in the United States alone (Shulman, 1993). There is no medical treatment to prevent or halt the progression of a cataract; nor is there any way to reverse a cataract once it has formed. The only known treatment is surgical. However, a medical treatment could be possible if we understand how a cataract forms and what makes it grow (Shulman, 1993). The ability of detecting a cataract at the earliest stages of formation will be useful in patient monitoring and in the development and in screening possible "anticataract" drugs or "diet" therapies. Kupfer of the National Eye Institute (NEI) of the National Institutes of Health (NIH) predicts that by the year 2000 new drugs will slow the progression of cataracts which is the leading cause of blindness worldwide (Long, 1992). Most recently Sardi (1995) has strongly argued in favor of using multivitamins, antioxidants, and nutritional supplements in eradicating cataracts. In-between the lens and the retina a gel-like fluid known as vitreous humor fills the rest of the eye globe. This fluid comprises $80 \%$ of the total volume of the eye and is the least understood component of an eye. Vitreous diseases can be detected by optically examining this fluid.

The technique of dynamic light scattering (DLS) or quasi-elastic light scattering (QELS) was first applied to the study of cataractogenesis in the pioneering work of Tanaka and Benedek (1976). An extensive review of using QELS to study cataracts has been given by Bursell et al (1990). DLS/QELS being a non-invasive and quantitative technique, seem to hold promising potential in its use as a routine ophthalmic device. But, its commercial scope as an ophthalmic diagnostic tool in clinical settings hitherto not materialized. This is mainly due to bulky equipment, optical alignment problems,

*National Research Council-NASA Research Associate at Lewis Research Center. 
polydisperse and interacting nature of the scattering species, and radiation exposure time. Since the original application of QELS, some twenty years ago by Tanaka and Benedek (1976), every subsequent study focused on rather easily accessible part (anterior chamber) of the eye i.e. the aqueous humor and the lens. Most recently Ansari and et al. (1995a and 1995b) in the USA and Rovati et al. (1995) in Switzerland reported the first experiments directed to the studies of the vitreous.

In the past few years DLS instrumentation has embraced several new and innovative technological advances. The immediate impact of these have resulted in the miniaturization of DLS instrumentation (1990). About four years ago a lens-less back-scatter fiber optic probe was developed by Dhadwal et al. (1991) to study concentrated particulate dispersions. Subsequently, Ansari et al. (1992) and Dhadwal et al. (1993) have shown the utility of this probe to the studies of cataractogenesis in excised bovine and cadaver eye lenses, and in live animals (Dhadwal, et al. 1995). Unfortunately these preliminary studies have certain limitations and pose severe constraints in probing different parts of the eye. Ansari and Suh (1995) recently developed a new DLS probe to study nucleation and aggregation phenomena during protein crystal growth in space (microgravity) experiments. Ansari et al. (1995) have also shown recently that this probe alleviates many major concerns discussed earlier when used as an ophthalmic diagnostics device and is much superior in performance when compared with the earlier reported work (Ansari, et al. 1992; Dhadwal, et al. 1993; 1995). The new probe provides accurate particle size determination of a variety of colloidal dispersions in 5 seconds at extremely low laser power levels. Furthermore, the major problem of probing different parts of the eye has been solved. In this report we briefly summarize some experimental in-vivo results on rabbits and a 3-D scan of an intact bovine eye lens.

\section{EXPERIMENTAL PROCEDURE AND SET UP}

The laser light from a laser diode is transmitted by a compact backscatter fiber optic probe to the eye. Depending upon the position of the scattering volume, Brownian motion of the particles in the aqueous humor, protein crystallines inside the lens and the macromolecules in the vitreous humor are monitored. The incident laser radiation interacts with the submicron particles dispersed in different regions of the eye. The particles scatter light and the intensity of this scattered light fluctuates in time due to the Brownian or thermal motion of the dispersed particles in the suspending medium. In a dynamic medium such as eye, when illuminated by laser light, a rapidly changing interference pattern due to the differences in the optical density of the scattering medium can be coherently detected by a DLS spectrometer. The rapidly fluctuating interference pattern at a photodetector contains information about the dynamics of the scattering medium and can be extracted by constructing a correlation function. A correlation function is constructed after detecting the scattered light by a photodetector and appropriate electronic processing via a amplifier-discriminator stage and a digital correlator. In the simplest case of dilute dispersions of spherical particles the slope of the correlation function provides quick and accurate determination of the particle's translation diffusion coefficient. The diffusion coefficient data can easily be transformed into average particle size, using Stokes-Einstein equation, provided the viscosity of the suspending medium, its temperature and refractive index are known.

A fiber optic probe comprising two monomode optical fibers and two GRIN micro lenses, as illustrated in Figure 1, provides a compact and remote means of studying the dynamical characteristics of the macromolecules in the eye. A $1.3 \mathrm{~cm}$ (diameter) $\times 2.3 \mathrm{~cm}$ (length) fiber optic probe contains the necessary optics to perform DLS measurements at a scattering angle of $161.5^{\circ}$. The probe is non-invasive and is conveniently positioned in front of the eye (cornea), but having no physical contact with any part of the eye. Two monomode optical fibers, each housed in a stainless steel ferrule, are mounted into a separate stainless steel housing. An air gap $(0-0.5 \mathrm{~mm})$ is intentionally left between the fiber housing and the lens housing in order to produce a tightly focused spot in the scattering volume. The two optical fibers in their housings are aligned and fixed into position off-axis with the GRIN lens. The two housings are placed inside a third (outer) housing made of stainless steel, and the back end of the housing is covered with a heat-shrink tubing. The two free ends of the optical fibers were terminated with FC/PC-type male connectors for easy mating with the laser diode and an avalanche photodiode detector (APD). The DLS data was analyzed using the commercial software provided by the Brookhaven Instruments Company of New York. 


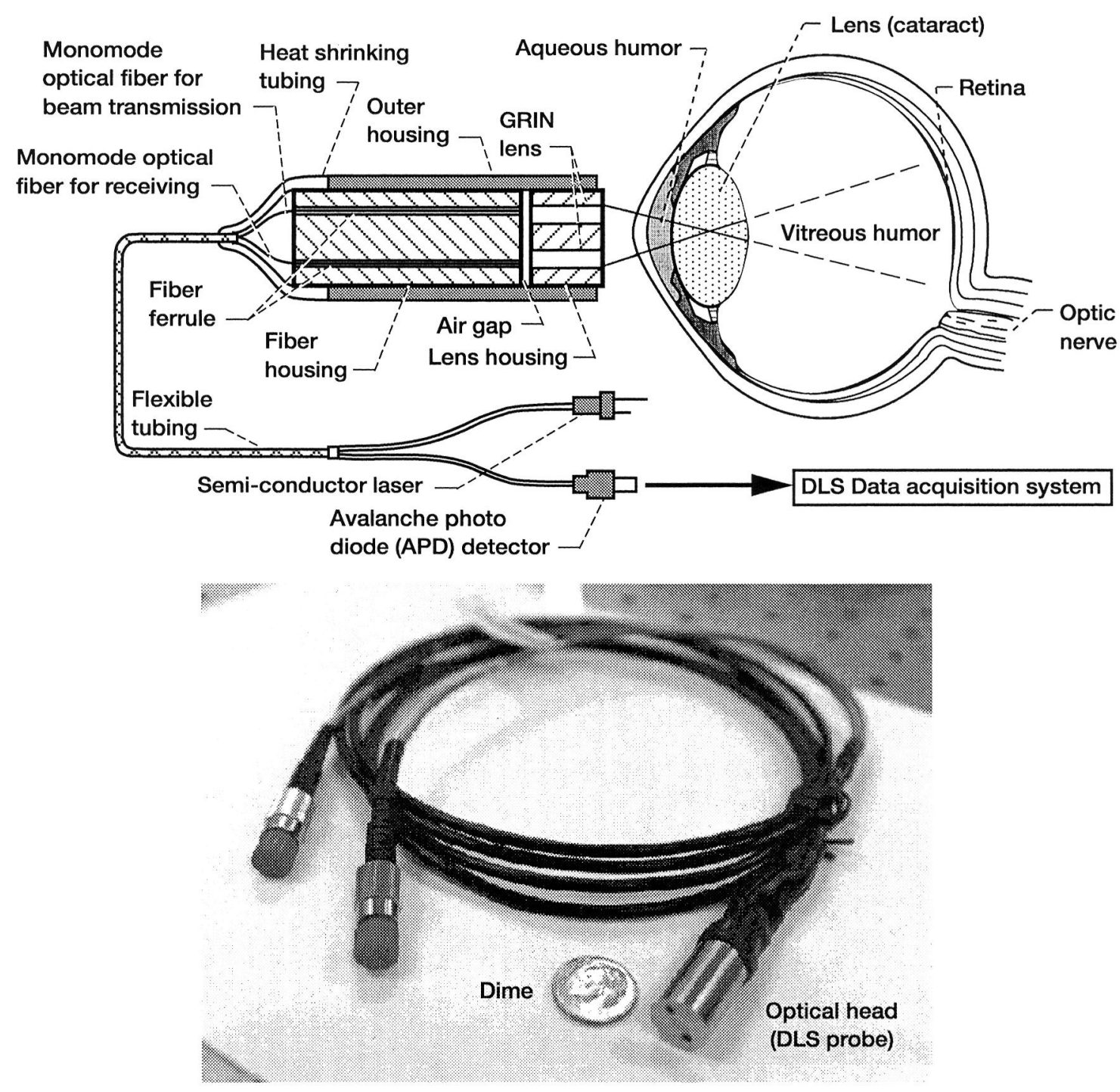

F|GURE 1. A Compact Device For Eye Diagnostics.

\section{RESULTS}

We report in-vivo DLS measurements on two live rabbits; one; 2 months old and the other; 6 months old. DLS measurements were performed on both left and right eyes as a function of the penetration depth from the corneal surface. This is shown in Figure 2. In the anterior chamber (aqueous humor) of the eye, we do not detect a considerable number of scattered photons and therefore conclude no presence of any substantially suspended particulate matter in this region. In a physical sense, $\alpha, \beta$, and $\gamma$ crystallines (proteins) constituting $~ 35 \mathrm{wt} . \%$ of the lens are dispersed in $65 \mathrm{wt} . \%$ water. Since $\alpha$-crystallines are the largest (molecular weight $\sim 1 \times 10^{6}$ daltons) they scatter most of the incident laser light. The measured $\alpha$-crystalline sizes are also similar in both left and right eyes. However, we find a specific trend as we move inside the lens and away from the anterior cortex. We see a gradual increase in the particle size as we move from the anterior cortex to the nucleus of the lens. Within the nucleus the size roughly remains constant, and then it gradually decreases as we move out of the nuclear region and into the posterior cortex. This result shows detailed structure of the 


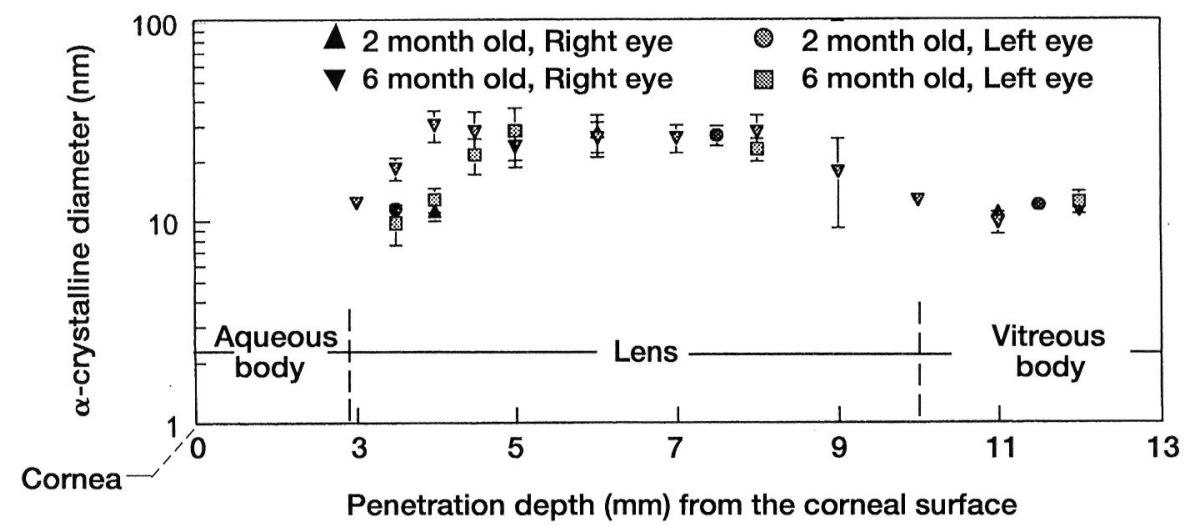

FIGURE 2. In-vivo DLS Results on Two Live Rabbits.

lens in terms of $\alpha$-crystalline size and its distribution in the lens tissue. A gradual increase in the size of $\alpha$-crystalline protein particles can be considered as an indication of a growing cataract. The $\alpha$-crystalline sizes reported here are reproducible within 5-10\%. In Figure 3 we present preliminary results identifying detailed crystalline structure of a transparent excised bovine eye lens. A 3-D plot is constructed after scanning the lens by the new fiber optic probe. This plot shows average protein size ( $\alpha$-crystalline + aggregates) as one moves from the anterior cortex to nucleus to posterior cortex inside the lens capsule. At the present time we are monitoring a group of laboratory rabbits for developing cataracts by feeding them different doses of naphthalene. This study will be reported elsewhere.

The vitreous humor of the eye described by Sebag (1989) is the least understood part of an eye. We present preliminary DLS measurements made in vivo. The vitreous body exhibits a two-exponential behavior, i.e. a fast and a slowly diffusing component, consistent with its gel-like properties. We see almost constant value for the fast component in this region. We ascribe this fast component due to the diffusion of hyaluronate molecular coils in water and the slow diffusion component due to the collagen-fibril network. A size distribution of the vitreous is presented in Figure 4. We calculated the molecular weight for the fast component to be $3.78 \times 10^{6}$ daltons. This is consistent with the range $\left(2-4.5 \times 10^{6}\right)$ of values given by Balazs and Delinger (1984). More in-vivo experimental work is being performed at this time in our laboratory to fully understand the structure of the vitreous humor.

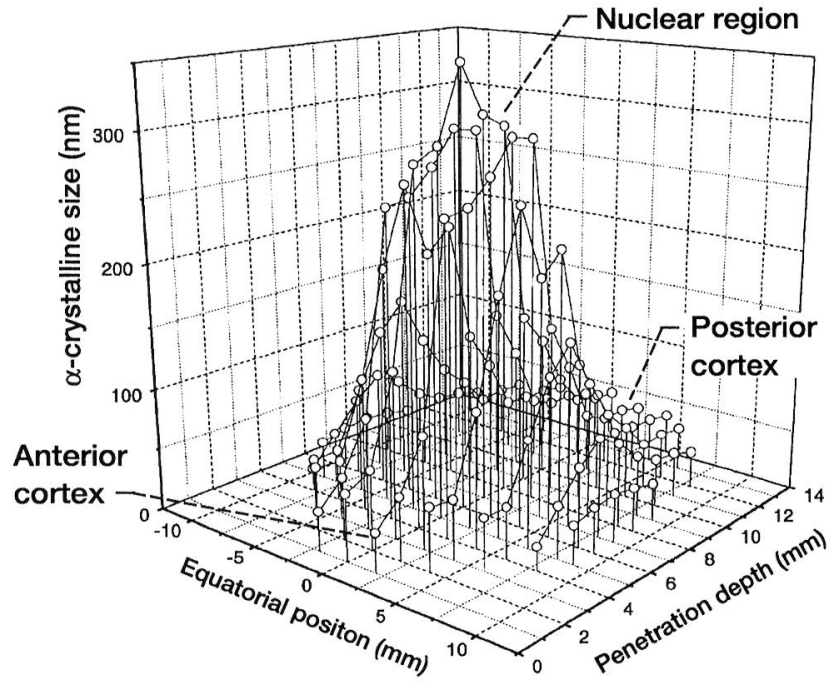

FIGURE 3. 3-D Scan of Protein Population in a Bovine Lens.

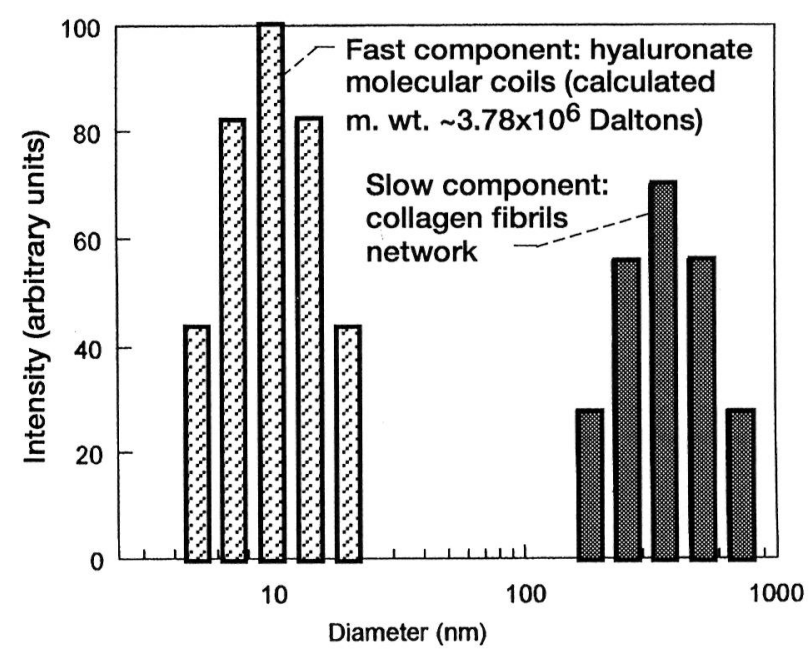

FIGURE 4. Six Month Old Rabbit Right Eye Posterior Chamber Vitreous Size Distribution (CONTIN Analysis) Penetration Depth From The Corneal Surface $11.0 \mathrm{~mm}$. 


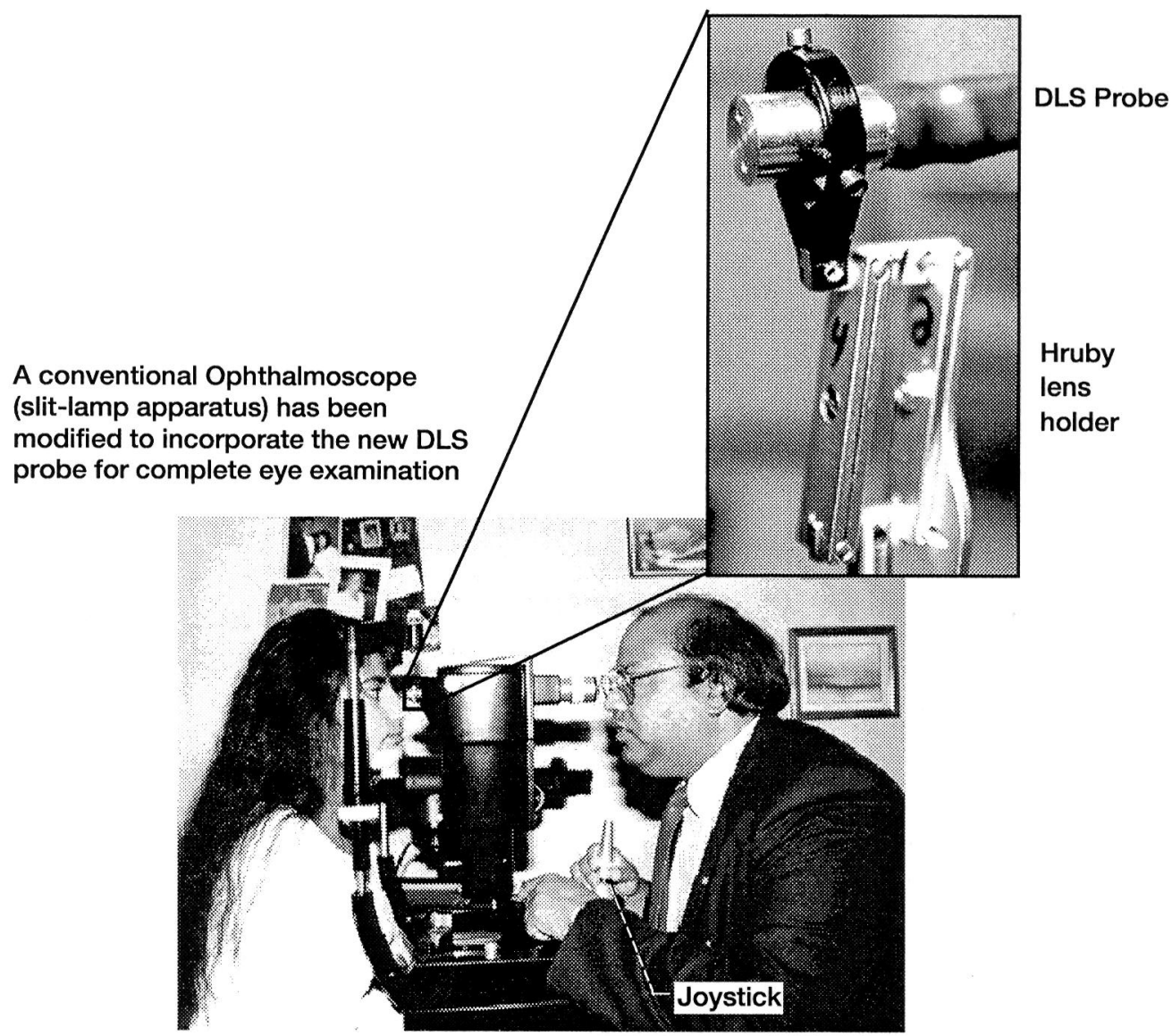

FIGURE 5. A Compact Device for Eye Diagnostics.

\section{CONCLUSION}

In this report we have briefly described a new DLS probe to non-invasively and quantitatively diagnose various parts of the eye. For clinical use we have mounted the DLS fiber optic probe on a Hruby lens holder. This compact assembly is then mounted on a slit-lamp apparatus as shown in Figure 5. The viewing area inside the eye can be controlled by using the joy stick. In addition to routine eye examination with a slit-lamp apparatus, our method provides early detection of cataracts, the characterization of hyaluronic acid chains and the collagen protein fibrils in the vitreous humor, and though not demonstrated here, the characterization of protein molecules and the metabolic waste product in the aqueous fluid in the anterior chamber of the eye. The patient correlation functions and the corresponding size distribution data has been integrated into OPIS ${ }^{\circledR}$ (Ophthalmic Patient Information System) computer software program. This facilitates in keeping complete patient profiles.

\section{Acknowledgements}

The authors are grateful to the Microgravity Science and Applications Division (MSAD), code UG of the NASA headquarters for supporting this research. This work was completed under a NASA grant NCC 166-3/CWRU. Kwang Suh wishes to thank NASA Lewis Research Center and the National Research Council for the award of a postdoctoral research fellowship. The cadaver eyes for this project were provided by Mr. Bob Littel of the Lions Eye Bank of Delaware Valley. 


\section{REFERENCES}

Ansari, R.R., Dhadwal, H.S., Campbell, M.C.W. and DellaVecchia, M.A. (1992) "A Fiber Optic Sensor for Ophthalmic Refractive Diagnostics," Proc. of Fiber Optic Medical and Fluorescent Sensors and Applications, January 23-24, 1992, Los Angeles, CA., SPIE Vol. 1648.

Ansari, R.R., Suh, K.I., DellaVecchia, M.A. and Dubin, S. (1995a) "Early Diagnosis of Cataracts Using a Fiber Optic System," in audio/video Proc. Cataract and Refractive Surgery: Current Issues, Session 2-B, ASCRS Symposium on Cataract, IOL and Refractive Surgery, April 1-5, 1995, San Diego, CA.

Ansari, R.R., Suh, K.I., DellaVecchia, M.A. and Dubin, S. (1995b) "Ophthalmic Diagnostics Using a New Dynamic Light Scattering Fiber Optic Probe," Proc. Medical Application, Conference on lasers in Ophthalmology III, SPIE, Bios Europe' 95, Barcelona, Spain, September 12-16, 1995, 2632(18).

Ansari, R.R. and Suh, K.I. (1995) "Sizing of Colloidal Particles and Protein Molecules in a Hanging Fluid Drop", Proc. Biomedical Optoelectronics in Clinical Chemistry and Biotechnology, SPIE, Bios Europe' 95, Barcelona, Spain, September 12-16, 1995. 2629(23).

Balazs, E.A. and Denlinger, J.L. (1984) "The Vitreous," in: The Eye, vol. IA, editor, H. Davson, Academic Press, 533-589.

Brown, J.G., Burnett, J., Mansbridge, C.I., Moir, C. (1990) "Miniature Laser Light Scattering Instrumentation for Particle Size Analysis," Appl. Opt. 29, 4159-4169.

Bursell, S.E., Magnante, P.C. and Chylack Jr., L.T. (1990) "In vivo Uses of Quasielastic Light Scattering Spectroscopy as a Molecular Probe in the Anterior Segment of the Eye," in Noninvasive Diagnostic Techniques in Ophthalmology, editor Barry R. Masters, Springer-Verglag, New York.

Dhadwal, H.S., Ansari, R.R., and Meyer, W.V. (1991) “A Fiber Optic Probe for Particle Sizing in Concentrated Suspensions," Rev. Sci. Instrum. 62(12), December 1991.

Dhadwal, H.S., Ansari, R.R. and DellaVecchia, M.A. (1993) "Coherent Fiber Optic Sensor for Early Detection of Cataractogenesis in a Human Eye Lens," J. Opt. Engineering, 32(2), February 1993.

Dhadwal, H.S., Ansari, R.R., DellaVecchia, M.A. and Dubin, S. (1995) "Fiber Optic System for In-vivo Sizing of Proteins in Animal Eye Lenses," Proc. Ophthalmic Technologies V, SPIE, 2393, 227-236, February 4-5, 1995, San Jose, CA.

Long, M.E. (1992) “The Sense of Sight,” in National Geographic, 182(5), Nov. 1992.

Rovati, L., Fankhauser,F. II, and Ricka, J. (1995) "Dynamic Light Scattering Spectroscopy of In-vivo Human Vitreous," Proc. Medical Application, Conference on Lasers in Ophthalmology III, SPIE, Bios Europe' 95, Barcelona, Spain, September 12-16, 1995, 2632(19).

Sardi, B. (1995) “Eradicating Cataracts," Townsend letter for doctors, June 1995.

Sebag, J. (1989) “The Vitreous: Structure, Function, and Pathobiology," Springer-Verlag, New York.

Shulman, J. (1993) "Cataracts: The Complete Guide from Diagnostics to Recovery for Patients and Families," St. Martins Press, New York.

Tanaka, T. and Benedek, G.B. (1976) "Observation of Protein Diffusivity in Intact Human and Bovine Lenses With Application to Cataract," Investigative Ophthalmology, 14(6), 449-456. 
Public reporting burden for this collection of information is estimated to average 1 hour per response, including the time for reviewing instructions, searching existing data sources, gathering and maintaining the data needed, and completing and reviewing the collection of information. Send comments regarding this burden estimate of any other aspect of this collection of Information, including suggestions tor redueing this burden, to Washington Headquarters Services, Directorate tor Information Operations and Reports, 1215 Jefferson Davis Highway, Suite 1204, Arlington, VA 22202-4302, and to the Office of Management and Budget, Papenwork Reduction Projed (0704-0188), Washington, DC 20503.

\begin{tabular}{|l|c|c|c|}
\hline 1. AGENCY USE ONLY (Leave blank) & $\begin{array}{r}\text { 2. REPOAT DATE } \\
\text { November } 1995\end{array}$ & $\begin{array}{r}\text { 3. REPORT TYPE AND DATES COVERED } \\
\text { Technical Memorandum }\end{array}$ \\
\hline
\end{tabular}

4. TITLE AND SUBTITLE

5. FUNDING NUMBERS

A Compact Fiber Optic Eye Diagnostic System

6. AUTHOR(S)

WU-963-45-0A

Rafat R. Ansari, Kwang I. Suh, Stephen Dubin, and Michael A. DellaVecchia

7. PERFoRMING ORGANIZATION NAME(S) AND ADDRESS(ES)

8. PERFORMING ORGANIZATION REPORT NUMBER

National Aeronautics and Space Administration

Lewis Research Center

Cleveland, Ohio 44135-3191

E-9986

9. SPONSORINGMONITORING AGENCY NAME(S) AND ADDRESS(ES)

10. SPONSORINGMONITORING AGENCY REPORT NUMBER

National Aeronautics and Space Administration

Washington, D.C. 20546-0001

NASA TM-107099

11. SUPPLEMENTARY NOTES

Prepared for the Space Technology and Applications International Forum sponsored by the NASA Technology Utilization Foundation, Albuquerque, New Mexico, January 7-11, 1996. Rafat R. Ansari, NASA Lewis Research Center; Kwang I. Suh, National Research Council-NASA Research Associate at Lewis Research Center; Stephen Dubin and Michael A. DellaVecchia, Drexel University, Biomedical Engineering and Science Institute, Philadelphia, Pennsylvania 19104. Responsible person, Rafat R. Ansari, organization code 6712, (216) 433-5008.

12a. DISTRIBUTIONAVAILABILTY STATEMENT

12b. DISTRIBUTION CODE

Unclassified - Unlimited

Subject Categories 35, 51, and 74

This publication is available from the NASA Center for Aerospace Information, (301) 621-0390.

13. ABSTRACT (Maximum 200 words)

A new fiber optic probe developed for determining transport properties of sub-micron particles in fluids experiments in a microgravity environment has been applied to study different parts of an eye. The probe positioned in front of an eye, delivers a low power $(\sim$ few $\mu \mathrm{W})$ light from a laser diode into the eye and guides the light which is back scattered by different components (aqueous humor, lens, and vitreous humor) of the eye through a receiving optical fiber to a photo detector. The probe provides rapid determination of macromolecular diffusivities and their respective size distributions in the eye lens and the gel-like material in the vitreous humor. In a clinical setting, the probe can be mounted on a standard slit-lamp apparatus simply using a Hruby lens holder. The capability of detecting cataracts, both nuclear and cortical, in their early stages of formation, in a non invasive and quantitative fashion, has the potential in patient monitoring and in developing and testing new drugs or diet therapies to "dissolve" or slow down the cataract formation before the surgery becomes necessary. The ability to detect biochemical and macromolecular changes in the vitreous structure can be very useful in identifying certain diseases of the posterior chamber and their complications, e.g., posterior vitreous detachment and diabetic retinopathy.

14. SUBJECT TERMS

Patient monitoring; Ophthalmic technologies; Gels; Dynamic light scattering; Cataracts; Fiber optic sensors

\begin{tabular}{l|l}
\hline 17. SECURTY CLASSIFICATION & 18. SECURTY CLASSIFICATION
\end{tabular} OF REPORT Unclassified OF THIS PAGE Unclassified

19. SECURITY CLASSIFICATION OF ABSTRACT Unclassified 